INFLAMMATORY BOWEL DISEASE

\title{
Safety and efficacy of intravenous pulse cyclophosphamide in acute steroid refractory inflammatory bowel disease
}

\author{
A Stallmach, B M Wittig, C Moser, J Fischinger, R Duchmann, M Zeitz
}

See end of article for authors' affiliations

Correspondence to:

Correspondence to:
Dr M Zeitz, Department of Internal Medicine I,

Benjamin Franklin Medical School, Free University of Berlin, Hindenburgdamm 30,12200 Berlin,

Germany; martin.zeitz

@medizin.fu-berlin.de

Accepted for publication 20 August 2002
Background and aims: One major problem in the management of steroid refractory attacks of patients with inflammatory bowel disease (IBD) is the establishment of a rapidly acting immunosuppressive regimen. Based on its well known efficacy in systemic vasculitis, intravenous cyclophosphamide pulse therapy was used in refractory IBD patients to evaluate both its efficacy and safety. Methods: Between December 1998 and May 2001, seven patients (Crohn's disease, n=5; indeterminate colitis, $n=1$ ) with severe steroid refractory IBD (Crohn's disease activity index (CDAl) 264-479 points) received 4-6 cycles of monthly treatment with intravenous cyclophosphamide $(750 \mathrm{mg})$ in a prospective uncontrolled pilot study.

Results: All patients improved after two intravenous pulses of cyclophosphamide and six of seven patients achieved complete remission (CDAl $<150$ points). One patient with Crohn's disease of the small and large bowel showed an impressive clinical response but did not enter into remission. Tapering to low dose steroids was possible in all responders. Remission was maintained in all patients for 18 months (median) but required a second course of intravenous pulse cyclophosphamide in one patient. The drug was well tolerated except for two episodes of candida oesophagitis.

Conclusions: Intravenous pulse cyclophosphamide may be a safe and effective treatment in patients with severe IBD unresponsive to steroid treatment and merits evaluation in a controlled trial.
T he natural course of the chronic inflammatory bowel diseases (IBD) Crohn's disease and ulcerative colitis is characterised by acute attacks requiring intensive medical care. The treatment of choice during severe attacks is high dose corticosteroids. ${ }^{12}$ However, $30-60 \%$ of patients develop a steroid refractory or dependent disease. ${ }^{3}$ Although immunosuppressive agents such as azathioprine, 6-mercaptopurine, cyclosporin, or humanised monoclonal antibodies directed against tumour necrosis factor $\alpha$ (TNF- $\alpha$ ) can be effective, a substantial number of patients are refractory even to combined use with glucocorticoids. For example, in a placebo controlled study, only $33 \%$ of patients with chronic treatment refractory Crohn's disease were in remission four weeks after a single infusion of anti-TNF- $\alpha$ antibodies (infliximab). Follow up showed that most patients did not remain in remission during the next 48 weeks if treatment with infliximab was not repeated. Finally, potential side effects such as prolonged immunosuppression with development of infectious diseases such as tuberculosis, development of autoantibodies against chimeric antibodies, and the cost of repeated applications of anti-TNF- $\alpha$ antibodies form the rationale for evaluation of other treatment options.

In addition, one unresolved problem in the treatment of severe IBD attacks is the availability of a rapidly acting and long lasting immunosuppressive regimen. Based on the established efficacy of intravenous cyclophosphamide in systemic vasculitis patients we used this agent in a severely ill 33 year old woman with steroid resistant indeterminate colitis who presented with fulminate bowel disease and extremely severe extraintestinal (skin) manifestations and fever (for details see results section: patient No 1). Analogous to a patient with gastrointestinal vasculitis in whom pulse cyclophosphamide was effective, ${ }^{5}$ our patient was treated by intravenous application of $750 \mathrm{mg}$ cyclophosphamide which induced a rapid clinical improvement of both the skin and bowel manifestations. After a second application of intravenous cyclophospha- mide four weeks later, a prolonged reduction in inflammatory activity with stable remission was observed.

This positive and impressive clinical course encouraged us to utilise intravenous pulse cyclophosphamide for steroid unresponsive acute attacks in patients with IBD and to prospectively assess its safety and efficacy in an open label study.

\section{PATIENTS AND METHODS}

Between December 1998 and May 2001, seven patients with acute (median Crohn's disease activity index (CDAI) 294 (range 284-479)) steroid refractory IBD (Crohn's disease $(n=6)$, indeterminate colitis $(n=1))$ participated in a prospective open label uncontrolled pilot study at the Department of Internal Medicine II, Saarland University. We included only patients with acute Crohn's disease or indeterminate colitis who did not respond to anti- inflammatory therapy with steroids (minimum of $50 \mathrm{mg}$ prednisolone) for more than seven days. This was the only selection criterium for this study. In addition, the majority of patients received parenteral nutrition due to the severity of their illness. Furthermore, six of seven patients had severe extraintestinal manifestations. However, this was not an inclusion criterium. In summary, these parameters indicate that patients were severely ill despite maximal standard therapy. All patients were diagnosed according to standard criteria by prior clinical assessment, radiology, endoscopy, and histology. Stool culture and serology ruled out concomitant infections. Patients were excluded from the study in the case of pregnancy or if renal or hepatic

Abbreviations: IBD, inflammatory bowel disease; TNF- $\alpha$, tumour necrosis factor $\alpha ; C D A I$, Crohn's disease activity index; WBC, white blood cell; SLE, systemic lupus erythematosus. 
Table 1 Patient characteristics

\begin{tabular}{|c|c|c|c|c|c|c|c|c|c|c|}
\hline $\begin{array}{l}\text { Patient } \\
\text { No }\end{array}$ & $\begin{array}{l}\text { Age/ } \\
\text { sex }\end{array}$ & Diagnosis & $\begin{array}{l}\text { Duration } \\
\text { of IBD (y) }\end{array}$ & Previous surgery & $\begin{array}{l}\text { Prednisolone } \\
\text { dose } \\
\text { (mg)/duration } \\
\text { (days) }\end{array}$ & CDAI & EIM & Localisation & $\begin{array}{l}\text { CDAl after } 3 \\
\text { applications }\end{array}$ & $\begin{array}{l}\text { Duration of } \\
\text { remission/clinical } \\
\text { course/follow up } \\
\text { (months) }\end{array}$ \\
\hline 1 & $23 / F$ & $C D$ & 10 & None & $60 \mathrm{iv} / 12$ & 294 & PG & $\mathrm{I}, \mathrm{C}, \mathrm{R}$ & 111 & $\begin{array}{l}29 / \text { relapse, then } \\
\text { second course }\end{array}$ \\
\hline 2 & $22 / F$ & $C D$ & 1 & None & $80 \mathrm{po} / 21$ & - & EN, arthritis & I, C & 102 & $35 /$ remission $/ 37$ \\
\hline 3 & $29 / M$ & IC & 16 & $\begin{array}{l}\text { Explorative } \\
\text { laparotomy and } \\
\text { doubleloop ileostomy }\end{array}$ & $50 \mathrm{po} / 21$ & 292 & PSC & $\mathrm{I}, \mathrm{C}, \mathrm{R}$ & 54 & $\begin{array}{l}20 / \\
\text { proctocolectomy } \\
\text { due to an } \\
\text { adenoma } / 38\end{array}$ \\
\hline 4 & $20 / \mathrm{F}$ & $C D$ & 3 & None & $100 \mathrm{iv} / 18$ & 290 & PSC & Stomach, C & 72 & $\begin{array}{l}\text { 18/relapse (steroid } \\
\text { treatment)/39 }\end{array}$ \\
\hline 5 & $19 / M$ & $C D$ & 2 & $\begin{array}{l}\text { Explorative } \\
\text { laparotomy }\end{array}$ & $60 / 50$ & 303 & None & J, I, C & 96 & $25 / 25$ \\
\hline 6 & $30 / \mathrm{F}$ & $C D$ & 19 & $\begin{array}{l}\text { Subtotal colectomy } \\
1984, \text { ileorectostomy } \\
1987,2 \text { anastomosis } \\
\text { resections 1988, } \\
1997\end{array}$ & 60 po/19 & 479 & Arthritis & I, C & 189 & $\begin{array}{l}\text { Clinical } \\
\text { improvement but } \\
\text { not remission }\end{array}$ \\
\hline 7 & $29 / F$ & $C D$ & 9 & $\begin{array}{l}\text { Sigmoid colon } \\
\text { resection 1997, } \\
\text { fistulae and abscess } \\
\text { drainage } 1997 \text {, } \\
1999,2000\end{array}$ & $80 \mathrm{iv} / 7$ & 264 & EN, arthritis & $\mathrm{I}, \mathrm{C}, \mathrm{R}$ & 0 & $\begin{array}{l}\text { 6/relapse after a } 5 \\
\text { month remission } \\
\text { period } \\
\text { (hemicolectomy)/ } \\
12\end{array}$ \\
\hline 1 * & $25 / F$ & $C D$ & 12 & None & $100 \mathrm{iv} / 21$ & 316 & None & $C, R$ & 69 & 12/remission/12 \\
\hline
\end{tabular}

$C D$, Crohn's disease; IC, indeterminate colitis; J, jejunum; I, ileum; $C$, colon; $R$, rectum; EIM, extraintestinal manifestation; PG, pyoderma gangrenosum;

EN, erythema nodosum; PSC, primary sclerosing cholangitis.

* Second course of cyclophosphamide application after flare up (see patients and methods).

- , Data not available.

impairment were present. Patient histories are summarised in table 1 . Written informed consent was given in advance.

Treatment with prednisolone $50 \mathrm{mg} /$ day or more for at least seven days had failed in all patients. Cyclophosphamide $750 \mathrm{mg}$ (ASTA Medica, Frankfurt, Germany) (independent of body weight) was given during a 12 hour hospital stay combined with intravenous fluids at a rate known to produce a urine output of $2 \mathrm{ml} / \mathrm{kg} / \mathrm{h}$. Mesna (2-mercaptoethansulphonic acid, Uromitexan (ASTA Medica, Germany), total dose equal to 50\% of the dose of cyclophosphamide to prevent haemorrhagic cystitis) was administered in three equal doses, during and after cyclophosphamide treatment, for 12 hours. Follow up white blood cell (WBC) counts with differential were obtained on post-cyclophosphamide days $7,10,14$, and 21 to detect each WBC nadir and rebound levels. Each patient received between four and six monthly treatments. All patients were continued on a daily regimen of prednisolone and the daily dose of prednisolone was decreased according to individual clinical activity. Azathioprine $(2.5 \mathrm{mg} / \mathrm{kg} / \mathrm{day})$ was initiated orally to maintain remission following cyclophosphamide treatment after the onset of remission. Video endoscopy of the large intestine, including the terminal ileum, was performed on the day before the first cyclophosphamide pulse and at weeks 4 and 8 .

CDAI was determined within one week before the first intravenous cyclophosphamide pulse and on weeks 2, 4, 8, and 12. Patients used a daily diary system before and during the study period for assessment of CDAI. In addition, a subjective score was assessed, as described by van Dullemen and colleagues. ${ }^{6}$ Excellent response was defined as 1 , good response as 2, fair response as 3, no response as 4 , and worsening as 5. One patient (patient No 1) relapsed after 26 months and received a second course of two doses of intravenous cyclophosphamide. During the 30 month study period all patients fulfilling the inclusion criteria were treated with cyclophosphamide.

\section{RESULTS}

\section{Case reports}

\section{Case No 1}

A 33 year old woman with an acute attack of extensive colitis showed only a partial response to systemic steroids $(60 \mathrm{mg}$ prednisolone/day) with regard to her bowel symptoms. Endoscopic examination revealed deep colonic and ileal ulcerations typical of Crohn's disease but histological examination of intestinal biopsies indicated features of ulcerative colitis. The patient's disease was classified as Crohn's disease. She developed extensive deep cutaneous ulcerations of the head and left leg (fig 1). Histological examination showed characteristics of pyoderma gangrenosum. Violation of the skin (for example, puncture for venous catheter) resulted in additional large deep ulcers. As an additional extraintestinal complication, septic temperatures above $40^{\circ} \mathrm{C}$ were observed. Laboratory diagnosis was consistent with severe inflammatory activity with strongly elevated $C$ reactive protein (286 $\mathrm{mg} / \mathrm{l}$ (normal range $0-5 \mathrm{mg} / \mathrm{l})$ ) and leucocytosis (16 200/ $\left.\mathrm{mm}^{3}\right)$. Extensive microbiological and virological workup revealed no signs of a local or systemic infection. As broad spectrum antibiotics in addition to steroid treatment were completely ineffective in controlling the systemic inflammatory reaction, and skin manifestations further deteriorated, immunosuppressive therapy with cyclophosphamide was introduced. Intravenous application of $750 \mathrm{mg}$ cyclophosphamide induced a rapid clinical improvement and reduction in inflammatory activity. Granulation of the cutaneous ulcer margins was visible by two weeks and clear evidence of healing occurred by one month. Five additional cycles of monthly treatment of intravenous cyclophosphamide $(750 \mathrm{mg}$ per cycle) were performed and the patient entered remission after the second cycle (course No 1, table 1). Video endoscopy of the large intestine revealed complete healing of colonic ulcerations after 12 weeks (fig 2A, B).

After the onset of remission, therapy with azathioprine (2.5 $\mathrm{mg} / \mathrm{kg}$ body weight) was started for remission maintenance. The patient had no symptoms for one year and made her own decision to stop azathioprine therapy. She developed a steroid refractory relapse 13 months later (course No 8, table 1) without extraintestinal symptoms, in particular no pyoderma gangrenosum occurred. At this time she again did not respond to prednisolone (100 mg/day intravenous) therapy but responded well to two cycles of intravenous cyclophosphamide. She is currently asymptomatic with azathioprine as maintenance therapy. 


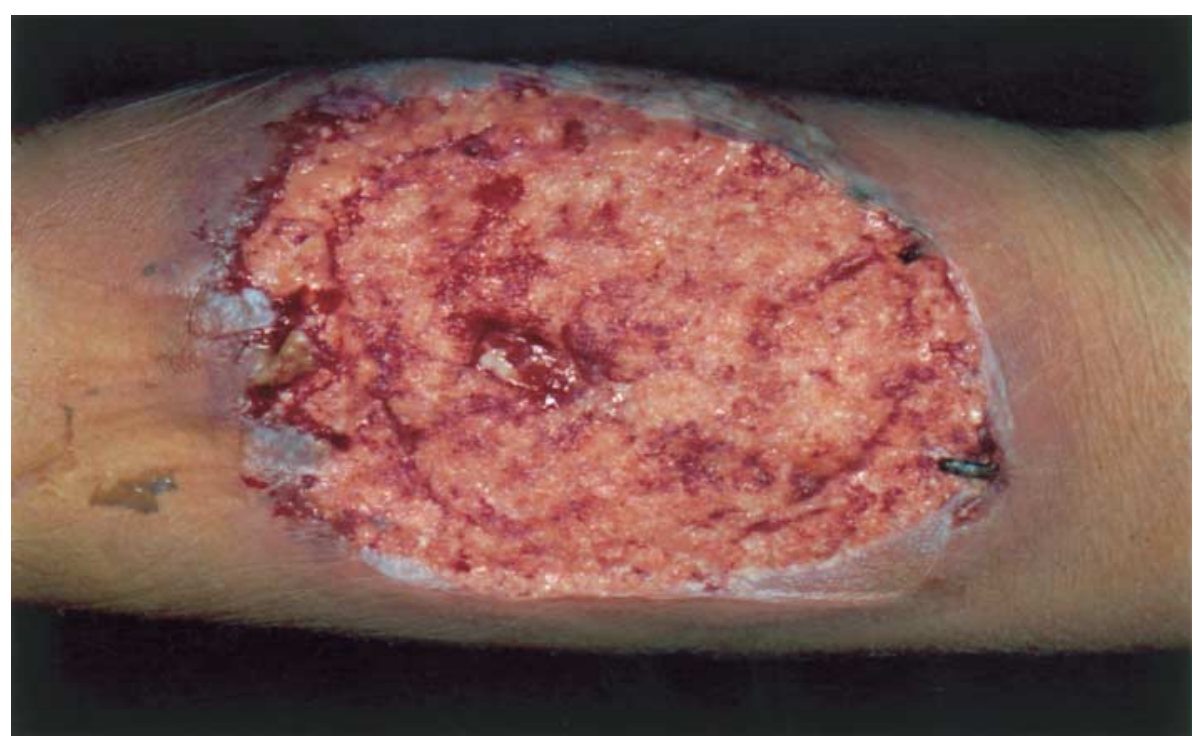

Figure 1 Pyoderma gangrenosum of the left leg in patient No 1.
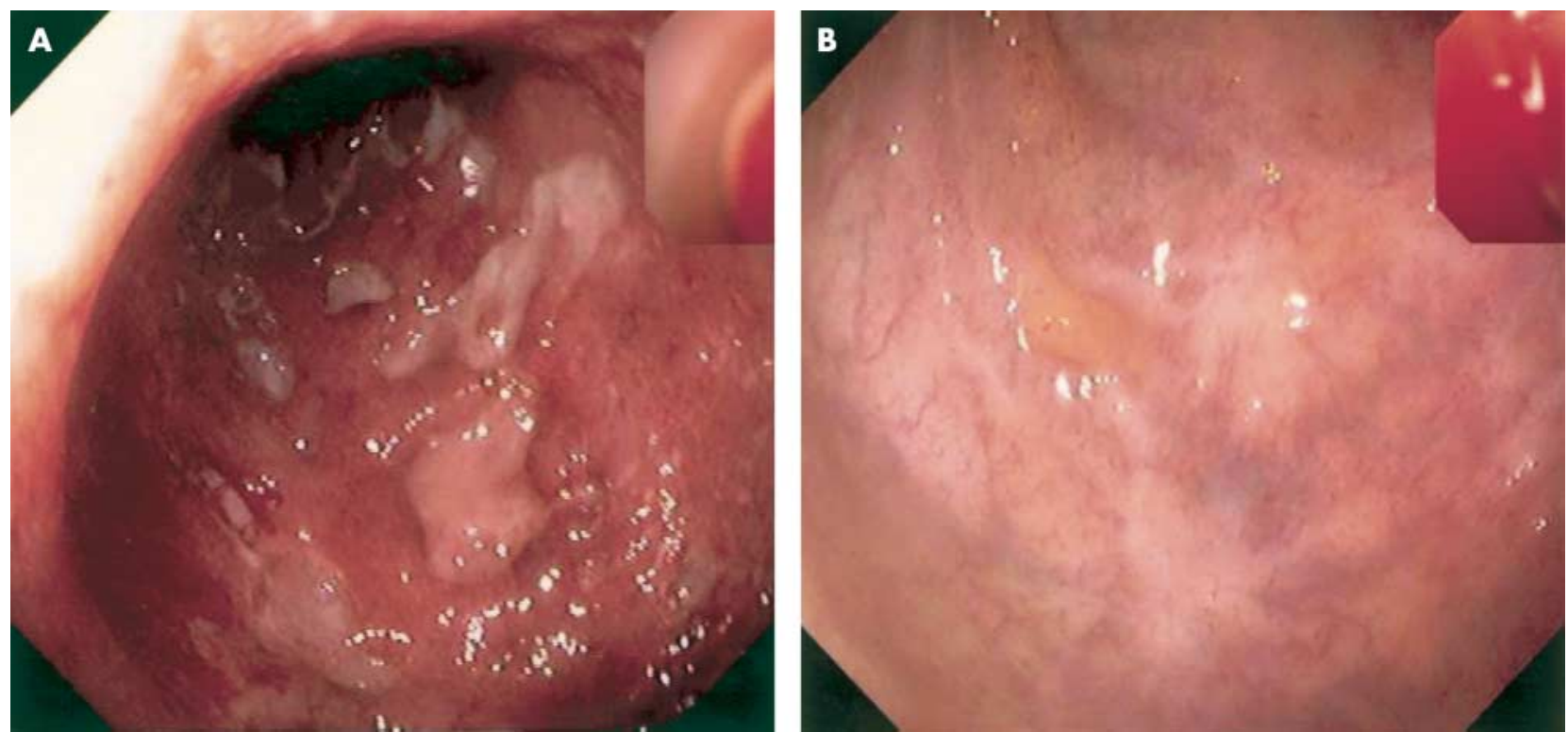

Figure 2 (A, B) Healing of colonic ulcerations after three cycles of cyclophosphamide in patient No 1.

\section{Case No 2}

Despite anti-inflammatory therapy with prednisolone $(60$ $\mathrm{mg}$ /day intravenous), this 22 year old woman with severe Crohn's ileocolitis developed massive haemorrhage requiring transfusion of 10 red blood cell concentrates within 48 hours. Minimum haemoglobin level was $4.8 \mathrm{~g} / \mathrm{dl}$. After transfer from a community hospital to our clinic, emergency colonoscopy revealed active bleeding from large and deep ulcerations in the colon and terminal ileum. To prevent proctocolectomy and resection of the terminal ileum, endoscopic clipping of bleeding ileal and colonic ulcerations was performed and immunosuppressive therapy with cyclophosphamide $(750 \mathrm{mg}$ per application) was initiated. After the first cyclophosphamide application, haemorrhage did not recur and the patient entered remission at week 4. Therapy with azathioprine was initiated and the patient is currently asymptomatic.

\section{Case No 3}

A 29 year old man with indeterminate colitis developed an acute abdomen with unremitting bloody diarrhoea and abdominal pain despite treatment with $60 \mathrm{mg} /$ day prednisolone. Explorative laparotomy revealed extensive deep ulcerations in the large and small bowel with perforations in the jejunum and ileum. A double loop ileostomy was created and intravenous pulse cyclophosphamide therapy was started on postoperative day 3. No clinical signs of infection (that is, fever or increase in $\mathrm{C}$ reactive protein) were observed. Clinical activity decreased after the first cyclophosphamide application and the patient entered remission after the second course of intravenous pulse cyclophosphamide. During follow up the patient was asymptomatic but colonoscopy after 20 months revealed a tubulovillous adenoma of the transverse colon which was not resectable by endoscopic treatment. Therefore, proctocolectomy was performed.

\section{Case No 4}

A 20 year old women with extensive Crohn's disease of the stomach and colon showed deterioration of her disease with increasing severe abdominal pain, vomiting, and diarrhoea (more than 20 trips to the toilet per day) despite receiving high dose prednisolone therapy and parenteral nutrition. Primary sclerosing cholangitis as an extraintestinal manifestation was present. This patient entered remission four weeks after the first cycle of intravenous pulse cyclophosphamide. 
No adverse events occurred. After six cycles of cyclophosphamide and initiation of azathioprine therapy, the patient remained in remission for 18 months. Then the patient developed a flare up of Crohn's disease which responded promptly to steroids.

\section{Case No 5}

A 19 year old man with extensive Crohn's disease of the small intestine developed severe abdominal pain. Steroid therapy in combination with azathioprine was stopped because of acute azathioprine related pancreatitis. Clinical activity response was poor after the first cyclophosphamide pulse but repeated application of cyclophosphamide induced long lasting remission after three months. Using 10-15 mg prednisolone per day, remission was maintained for 25 months until the present.

\section{Case No 6}

This 30 year old woman with extensive Crohn's disease of the small and large intestine had severe abdominal pain and chronic anaemia (haemoglobin $8.1 \mathrm{~g} / \mathrm{dl}$ ) as major indicators of disease activity. The patient's CDAI responded to intravenous pulse cyclophosphamide therapy and decreased from 479 to 157 points. After five courses of intravenous pulse cyclophosphamide, therapy was stopped as the patient did not enter into stable remission. She later showed no response to one infusion of anti-TNF monoclonal antibodies (infliximab) $(5 \mathrm{mg} / \mathrm{kg}$ body weight) and had to undergo proctocolectomy.

\section{Case No 7}

A 29 year old woman presented with abdominal pain, diarrhoea, and a painful draining perianal fistula which had not responded to prior infusion of anti-TNF monoclonal antibody (infliximab) ( $5 \mathrm{mg} / \mathrm{kg}$ body weight, three applications) and azathioprine therapy $(3.5 \mathrm{mg} / \mathrm{kg}$ body weight). Endoscopic examination and NMR tomography revealed transmural inflammation of the left colon with deep ulcerations and blind fistulas into the adjacent tissue. Clinical activity decreased after the first cyclophosphamide application and the patient entered into remission before the second course of intravenous pulse cyclophosphamide was performed. Five cycles were performed and the patient went into remission. However, six weeks after the last cyclophosphamide application she developed abdominal pain. Colonoscopy revealed a fistula in the left colon and a surgical hemicolectomy was performed.

\section{Clinical response}

All patients still had severe disease activity and/or life threatening extraintestinal manifestations during the initial high dose steroid phase, which were the main inclusion criteria for the study. After the first cyclophosphamide intravenous pulse, all patients noted a substantial reduction in stool frequency. Median CDAI was 294 before treatment and decreased to 139 at week 4,121 at week 8, and 111 at week 12 (fig 3). Extraintestinal manifestations of IBD responded dramatically after treatment with pulse cyclophosphamide. Pyoderma gangrenosum in patient No l (see fig 1) improved substantially and arthritis and/or arthralgia, if present at baseline (see table $1)$, vanished in all patients after treatment.

Related to the decrease in clinical activity, reduction of steroids was possible in all patients and the median prednisolone dose after 12 weeks (that is, after three intravenous pulse cyclophosphamide doses) was $10 \mathrm{mg} /$ day (range 10-15). However, discontinuation of corticosteroid treatment was not performed during the first six months after initiation of intravenous pulse cyclophosphamide therapy.

In seven treatment courses (and one retreatment in the relapsing patient), all patients reported improvement of subjective symptoms within two weeks after initiation of intravenous pulse therapy. Four weeks after the first applica-

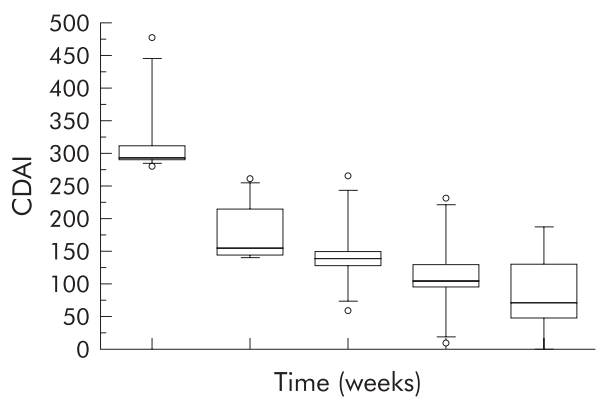

Figure 3 Crohn's disease activity index (CDAl) after initiation of intravenous pulse cyclophosphamide therapy ( $n=7)$. Values represent medians, bars represent 25 th and 75 th percentiles, and error bars indicate the 5th and 95th percentiles. Patient No 3 underwent a double loop ileostomy and therefore CDAl could not be calculated during follow up.

tion, six of seven patients scored an "excellent or good response to treatment" ( score 1) on the subjective scoring list.

\section{Side effects}

Intravenous pulse cyclophosphamide was well tolerated in all patients. Prior to the initiation of treatment with cyclophosphamide, mean WBC was 15500 cells $/ \mathrm{mm}^{3}$ (range 8300 to 21100 cells $\left./ \mathrm{mm}^{3}\right)$. After each monthly treatment, the mean nadir WBC count for all courses of pulse cyclophosphamide was 11400 cells $/ \mathrm{mm}^{3}$ (range 1700 to 23800 cells $/ \mathrm{mm}^{3}$ ). The lowest nadir of WBC counts occurred in one patient who received azathioprine in a high dose ( $3.5 \mathrm{mg} / \mathrm{kg}$ body weight) which was started 1.5 years prior to cyclophosphamide treatment. Four weeks after each pulse of cyclophosphamide and prior to each additional pulse treatment, WBC counts returned to normal. Lymphocyte subset distribution in all patients did not differ significantly from control subjects. In addition, there were no significant changes in relative $\mathrm{T}$ and $\mathrm{B}$ cell numbers in peripheral blood.

Two patients developed symptoms of candida oesophagitis during the first or second intravenous pulse cyclophosphamide treatments which responded promptly to oral ketoconazole. A third patient presented symptoms of an uncomplicated urinary tract infection.

\section{DISCUSSION}

In patients with IBD, the incidence of steroid refractory episodes is low. Nevertheless, these episodes represent a difficult therapeutic challenge. Approximately $12-20 \%$ of cases may be steroid resistant and potential candidates for bowel resection. ${ }^{2}$ However, because surgery may result in short bowel syndrome or in loss of the entire colon, alternative strategies are desirable. Based on previous observations of improvement in autoimmune diseases, we assessed the safety and efficacy of cyclophosphamide pulse therapy in steroid unresponsive attacks of patients with fulminant IBD. Although the present study is small and uncontrolled, we observed complete clinical remission in all but one patient in this subgroup of patients with severe IBD. After the first pulse of cyclophosphamide, CDAI decreased in all patients, and after the second pulse six patients went into long lasting remission (CDAI $<150$ points, see fig 3). The number of cyclophosphamide pulses is an important point of debate. In our study, onset of remission was observed after one or two pulses. Then, we started maintenance therapy with azathioprine. If maintenance therapy with azathioprine is planned, usually a period of 2-6 months has to be bridged before the drug starts to act. ${ }^{7}$ Therefore, we continued intravenous cyclophosphamide pulse therapy for 5-6 months but further trials are needed to address this issue. Less frequent applications (for example, 2-4 pulses) might be sufficient. Using this concept we achieved a median remission 
period of 18 months (range 3-29) in our subgroup of patients with severe IBD, usually characterised by their poor prognosis.

Cyclophosphamide is a potent suppressor of immune function, at high doses resulting in a sustained decrease in both the number and function of T and B cells. ${ }^{8}$ It is an effective therapy in certain autoimmune diseases. However, its efficacy varies For example, in severe systemic lupus erythematosus $(\text { SLE })^{910}$ and various forms of systemic vasculitis such as Wegener's granulomatosis or polyarteritis nodosa, its efficacy has been proved in several studies. Furthermore, intravenous cyclophosphamide appears to be effective in controlling the lesions of pyoderma gangrenosum and inducing remission for a substantial period in many individuals. ${ }^{11}$ On the other hand, in rheumatoid arthritis (except rheumatoid vasculitis) and in giant cell arteritis, cyclophosphamide is less effective. ${ }^{12}$ Based on these inconsistent findings, the efficacy of cyclophosphamide in severe IBD is not predictable. Published experiences with cyclophosphamide in severe IBD are very limited. One report described a clinical response to cyclophosphamide and epirubicin in a female with breast cancer, intractable Crohn's colitis, and perianal disease..$^{14}$ In another study the usefulness of cyclophosphamide in patients with Crohn's disease and azathioprine induced pancreatitis was studied. Deterioration in disease activity was observed after initiation of low oral daily cyclophosphamide therapy in three of four patients with severe Crohn's disease. ${ }^{15}$ These conflicting results clearly warrant a more systematic approach to the efficacy and safety of cyclophosphamide in IBD patients.

The remarkable clinical benefit of cyclophosphamide therapy in our study was obtained in the absence of relevant toxicity or side effects. We used an intermittent high dose intravenous cyclophosphamide pulse regimen as it has been shown in severe SLE that pulse therapy had at least comparable efficacy and less toxicity than treatment with daily low dose cyclophosphamide. ${ }^{16}{ }^{17}$ It should be noted that this therapeutic regimen caused no septic complications in our severely ill patient group with major bowel ulcerations. For example, in patient No 3, explorative laparotomy revealed extensive transmural ulcerations with penetration in the jejunum and ileum. Intravenous pulse cyclophosphamide therapy was started in this patient on postoperative day 3 and no clinical signs of infection (for example, fever or increase in $\mathrm{C}$ reactive protein) were observed. In common with other patients, clinical activity decreased after the first cyclophosphamide application and this patient entered into remission after the second course of intravenous pulse cyclophosphamide. Two other patients developed signs of candida oesophagitis after the first or second course of cyclophosphamide which were controlled by adequate treatment. In addition, one patient developed symptoms of an uncomplicated urinary tract infection.

Prolonged use of daily cyclophosphamide has been associated with toxic effects such as gonadal dysfunction (approximately 70\%), bone marrow suppression, haemorrhagic cystitis (17-34\%), and increased risk of neoplasms. ${ }^{17-19}$ In studies of patients with SLE, Balow et al have demonstrated that intermittent pulse cyclophosphamide in SLE was at least as efficacious as daily therapy while utilising lower cumulative doses and producing less toxicity. ${ }^{16}$ Haemorrhagic cystitis or malignancy has not been noted in any of their patients receiving pulse cyclophosphamide over an observation period now extending into its second decade..$^{20}$

Antibodies to TNF- $\alpha$ such as infliximab have been used in patients with active Crohn's disease who were resistant to standard therapy. ${ }^{4}$ It should be mentioned that this drug clearly has limitations in its efficacy as only $33 \%$ of patients obtain a stable clinical remission, and long term treatment strategies are poorly defined. It should also be noted that data on the long term side effects of infliximab are still spare and recent reports on infectious complications such as tuberculosis are appearing. Cyclophosphamide has been used for more than 20 years in autoimmune diseases, hence side effects are more predictable. Furthermore, administration of cyclophosphamide is standardised and costs are low (approximately $€ 100$ for one application of $750 \mathrm{mg}$ ).

In conclusion, treatment with pulse cyclophosphamide caused no major adverse events or side effects. Most patients entered into remission after two cyclophosphamide pulses. Based on these initial experiences, cyclophosphamide may be a good option to bridge the gap before the effect of azathioprine (or 6-mercaptopurine) starts. Furthermore, cyclophosphamide may help reduce the need for high dose steroids more rapidly in the initial treatment phase and therefore minimise the well known side effects, especially at high doses. Our pilot study has shown that cyclophosphamide doses of less than 3-6 g are sufficient to induce remission and bridge the gap before the effect of azathioprine starts. This dose of cyclophosphamide is known to have only minor long term toxicity. This pilot study thus indicates that pulse cyclophosphamide treatment has clinical potential in a subgroup of patients with severe steroid resistant IBD. Controlled studies to further evaluate the clinical efficacy of intravenous pulse cyclophosphamide are therefore warranted.

\section{ACKNOWLEDGEMENT}

The BMBF competence network programme "Chronic inflammatory bowel disease" supported this work. We are indebted to the medical endoscopy nurses, and the staff of M201, M202, and M203 for excellent care of patients. We thank Professor J Sieper for critical discussion of the manuscript.

\section{Authors' affiliations}

A Stallmach, B M Wittig*, C Moser, J Fischinger, R Duchmann*, M Zeitz*, Department of Internal Medicine II, Saarland University, Homburg, Germany

*Present address: Department of Internal Medicine I, Benjamin Franklin Medical School, Free University of Berlin, Berlin

\section{REFERENCES}

1 Summers RW, Switz DM, Sessions JT Jr, et al. National Cooperative Crohn's disease study: results of drug treatment. Gastroenterology 1979;77:847-69.

2 Malchow H, Ewe K, Brandes JW, et al. European Cooperative Crohn's Disease Study (ECCDS): results of drug treatment. Gastroenterology 1994:86:249-66.

3 Munkholm P, Langholz E, Davidsen $M$, et al. Frequency of glucocorticoid resistance and dependency in Crohn's disease. Gut 1994; $35: 360-2$.

4 Targan SR, Hanauer SB, van Deventer SJH, et al. A short-term study of chimeric monoclonal antibody $\mathrm{CA} 2$ to tumor necrosis factor alpha for Crohn's disease. Crohn's Disease cA2 Study Group. N Engl J Med 1997:337:1029-35.

5 Grimbacher B, Huber M, von Kempis J, et al. Successful treatment of gastrointestinal vasculitis due to systemic lupus erythematosus with intravenous pulse cyclophosphamide: a clinical case report and review of the literature. Br J Rheumatol 1998;37:1023-8.

6 van Dullemen HM, van Deventer SJ, Hommes DW, et al. Treatment of Crohn's disease with anti-tumor necrosis factor chimeric monoclonal antibody (cA2). Gastroenterology 1995:109:129-35.

7 Present DH, Korelitz BI, Wisch N, et al. Treatment of Crohn's disease with 6- mercaptopurine. A long-term, randomized study. N Engl J Med 1980;302:981-7

8 Cupps TR, Edgar LC, Fauci AS. Suppression of human B lymphocyte function by cyclophosphamide. J Immunol 1982;1 28:2453-7.

9 Illei GG, Austin HA, Crane $M$, et al. Combination therapy with pulse cyclophosphamide plus pulse methylprednisolone improves long-term renal outcome without adding toxicity in patients with lupus nephritis. Ann Int Med 2001;135:248-57.

10 Takada K, Illei GGB. Cyclophosphamide for the treatment of systemic lupus erythematosus. Lupus 2001;10:154-61.

11 Reynoso-von Drateln C, Perla-Navarro AV, Gamez-Nava Jl, et al. Intravenous cyclophosphamide pulses in pyoderma gangrenosum:an open trial. J Rheumatol 1997:24:689-93.

12 Keysser G, Keysser C, Keysser M. Treatment of refractory rheumatoid arthritis with low-dose cyclophosphamide. Long-term follow-up of 108 patients. Z Rheumatol 1998;57:101-7.

13 Suarez-Almazor ME, Belseck E, Shea B, et al. Cyclophosphamide for rheumatoid arthritis. Cochrane Database Syst Rev 2000;CD001157.

14 Rieger N, Stahl J, Wattchow D. Intractable Crohn's colitis and perianal disease responding to cyclophosphamide and epirubicin. Dig Dis Sci 1997;42:2367-9 
15 Nyman M, Hansson I, Eriksson S. Long-term immunosuppressive treatment in Crohn's disease. Scand J Gastroenterol 1985:20:1197-203.

16 Balow JE, Austin HA, Muenz LR, et al. Effect of treatment on the evaluation of renal abnormalities in lupus nephritis. N Engl J Med 1984;311:491-5

17 Austin HA, Klippel JH, Balow JE, et al. Therapy of lupus nephritis. Controlled trial of prednisone and cytotoxic drugs. N Engl J Med 1986;313:614-19.
18 Boltus JAM, Boersma JW, Hartman AP, et al. The occurence of malignancies in patients with rheumatoid arthritis treated with cyclophosphamide. Ann Rheum Dis 1983;42:368-73.

19 Baker GL, Kahl LE, Zee BE. Malignancy following treatment of rheumatoid arthritis with cyclophosphamide. Am J Med 1989;83:1-9.

20 Hoffman GS, Leavitt RY, Fleisher TA, et al. Treatment of Wegener's granulomatosis with intermittent high-dose intravenous cyclophosphamide. Am J Med 1990;89:403-10.

政

\section{Have your say}

\section{eLetters}

If you wish to comment on any article published in Gut you can send an eLetter using the eletters link at the beginning of each article. Your response will be posted on Gut online within a few days of receipt (subject to editorial screening).

www.gutjnl.com 